\title{
LAS DENOMINACIONES DE ORIGEN EN LA ACTUAL LEGISLACIÓN. PROPUESTAS PARA SU COMPLEMENTO Y ACTUALIZACIÓN ${ }^{1}$
}

\author{
Eduardo Jequier Lehuedé2 \\ Rafael Areyuna Navarro ${ }^{3}$
}

SUMARIO: Introducción. 1).- Tratamiento en la legislación vigente. 2). - Propuestas ante modificaciones legales. A) Reglamento de la denominación de origen. (a) Determinación precisa del producto amparado por la denominación. (b) Delimitación de la zona de producción. (c) Variedades o razas aptas para producir la materia prima. (d) Procedimientos o prácticas de producción. (e) Producto final. ( $f$ ) Registros. ( $g$ ) Control. (b) Derechos y obligaciones de los inscritos en la denominación. (i) Establecimiento de un Consejo Regulador. (j) Infracciones y sanciones. B) Consejo Regulador de la Denominación de Origen. (a) Naturaleza jurídica. (b) Estructura interna. (c) Integración. (d) Funciones. i. - Control de producción. ii.- Velar por el prestigio de la denominación. iiii.Certificación de productos amparados por la denominación. iv. - Gestión de recursos de la denominación. v. - Representación de la asociación. 3). - Denominaciones en el norte de Chile. A) Queso de Huentelauquén. B) Aceitunas del Huasco. C) Limones de Pica. D) Aceitunas de Azapa.

RESUMEN: El presente trabajo trata sobre el análisis del estado actual de las denominaciones de origen en la legislación nacional. Propone algunos interesantes puntos que debieran ser considerados para precisar el tema de la regulación de las denominaciones de origen. Finalmente, se concluye con la identificación de ciertos productos del norte de Cbile que indudablemente ban de proyectarse como futuras denominaciones de origen.

Palabras clave: Denominación de origen - Producto - Raza - Zona de producción - Consejo regulador.

\footnotetext{
1 El presente trabajo corresponde a un extracto de la investigación que los autores desarrollaron con el apoyo de la Dirección General de Investigación y Cooperación Técnica, de la Universidad Católica del Norte, marco de Proyectos de Investigación Científica y Tecnológica seleccionados por dicha entidad el año 2002. Profesor de Derecho Económico, Escuela de Derecho, Universidad Católica del Norte, sede Coquimbo.

3 Abogado. Profesor de Introducción al Derecho y de Derecho Civil, Escuela de Derecho, Universidad Católica del Norte, sede Coquimbo.
} 


\section{INTRODUCCIÓN}

El trabajo aquí presentado corresponde al capítulo 4 del proyecto denominado "La denominación de origen como un instrumento de protección e identificación de productos agroindustriales", tema que en relación con el pisco chileno fuera analizado en esta misma revista bajo el concepto de una versión de tesis resumida.

El capítulo que pasamos a exponer hace un análisis del estado actual de las denominaciones de origen en la legislación nacional y, ante la inminencia de cambios legislativos en la materia, propone algunos interesantes puntos que debieran ser considerados para precisa regulación del tema de las denominaciones de origen.

Esta selección termina con la identificación de ciertos productos del norte de Chile que indudablemente han de proyectarse como futuras denominaciones de origen.

\section{TRATAMIENTO EN LA LEGiSlación Vigente}

La regulación nacional en materia de denominaciones de origen ha sido hasta hoy confusa, sin definiciones conceptuales concretas y con el uso recurrente de terminología vaga e imprecisa. Además de estas deficiencias formales, por cierto no menores, el tratamiento que ha dado el legislador al tema se ha caracterizado también por la falta de un sentido de unidad entre las diversas normas dictadas sobre la materia, de manera que no ha sido posible crear una cultura en relación con las denominaciones de origen, amén de no existir una institucionalidad particular en relación con estas materias. Asimismo, las normas dictadas al respecto han estado circunscritas de manera prácticamente exclusiva a la actividad vitivinícola, descartando la aplicación de las denominaciones de origen a otras actividades.

El resultado de esta deficiente regulación normativa se traduce en que numerosos productos no cuentan en la actualidad con esta importante herramienta de protección y promoción productiva y de comercio, lo que impone un cambio profundo en el tratamiento y definitivo reconocimiento de esta materia en cuanto fenómeno jurídico relevante para el desarrollo productivo del país y, más aún, para su inserción definitiva en mercados externos -como el europeo- que presentan un desarrollo considerable en la protección y regulación de las denominaciones de origen.

\section{PROPUESTAS ANTE MODIFICACIONES LEGALES}

Ante el panorama arriba descrito, el Ejecutivo ha enviado al Honorable Congreso Nacional un proyecto que pretende reformar la Ley 
19.039, que establece las normas aplicables a los Privilegios Industriales y protección de los derechos de Propiedad Industrial. El objetivo general de dicho proyecto es ajustar el tratamiento de las materias que son objeto de dicha ley a las normativas internacionales que sobre el punto han sido establecidas en acuerdos de comercio suscritos por Chile y, específicamente, con aquellas regulaciones y directrices emanadas de la Organización Mundial de Comercio -OMC-, ${ }^{4}$ por cierto más completas y precisas que la normativa actualmente existente en nuestro país.

Sobre la base de dicho proyecto y, además, desde la perspectiva de los requerimientos efectivos de un sistema de denominaciones de origen, pensamos que una regulación seria sobre el tema implica normar al menos lo concerniente al reglamento de la denominación y a su estructura orgánica de autorregulación y control, según pasamos a explicar.

\section{A) Reglamento de la denominación de origen}

Conjuntamente con la normativa general y abstracta contenida en una ley u otra norma de tal carácter, es necesario que las denominaciones de origen tengan una regulación particular que rescate las peculiaridades de cada producto en relación con su entorno geográfico, lo que en la mayoría de los ordenamientos ha adoptado la forma de un reglamento interno para cada denominación.

En este sentido, el proyecto de modificación de la Ley $\mathrm{N}^{\circ} 19.039$ prescribe que la solicitud de registro de una denominación de origen debe ser acompañada de un proyecto de reglamento específico de uso y control de la denominación solicitada, precisando en consecuencia que las denominaciones se regirán por la Ley $\mathrm{N}^{\circ} 19.039$ y por los reglamentos particulares de cada denominación.

En cuanto a su contenido, el reglamento de las denominaciones de origen habrá de consignar la normativa particular que sobre la materia se hayan dado los titulares de cada denominación, lo que hace especialmente relevante -por ende- la adecuada organización interna de los mismos y la representatividad que logren sobre el total de productores dentro de la misma actividad económica. En este sentido, entonces, la norma debe establecer al menos las bases o parámetros mínimos de autorregulación y representatividad, cuestión que hasta ahora no contempla el proyecto de reforma tantas veces indicado.

Refiriéndose al rol de los reglamentos en las denominaciones de origen, Botana Agra estima que "el reglamento particular representa el más alto grado de concreción de las normas aplicables a cada Denomina-

4 Con fecha 17 de mayo de 1995 se publicó el Decreto $\mathrm{N}^{\circ} 16$ del Ministerio de Relaciones Exteriores, que promulgó el acuerdo de Marrakech, por el que se establece la Organización Mundial del Comercio-OMC-y los Acuerdos Anexos 1A, 1B, 1C, 2 y 3. 
ción de Origen; y sin perjuicio de ello, constituye al propio tiempo la norma institucional básica de la Denominación, pues en él se regulan aspectos que van desde los estrictamente organizativos, pasando por los de carácter jurídico-material, hasta los meramente técnicos ${ }^{5 "}$.

Atendida la importancia del reglamento de las denominaciones de origen y siguiendo las pautas entregadas por el precitado autor, ${ }^{6}$ estimamos que todo reglamento debe tener un contenido mínimo, siendo por lo mismo -y según se dijo más arriba- misión de la norma legal el establecer el marco general que debe ser completado en cuanto a su contenido específico por los respectivos usuarios de cada denominación.

Según lo expuesto, a nuestro entender los reglamentos de las denominaciones de origen nacionales deberían considerar al menos los siguientes aspectos:

\section{a) Determinación precisa del producto amparado por la denominación}

Es indispensable que cada reglamento especifique de la mejor forma posible los productos que serán objeto de la protección ofrecida por la denominación. Pensamos que esta determinación debe ser hecha hasta el punto que no quede duda razonable que lleve a la confusión respecto del producto objero de la denominación. La determinación de la mayor o menor descripción de los productos amparados debe ser una cuestión entregada a los propios titulares interesados.

\section{b) Delimitación de la zona de producción}

Este punto debe considerar toda el área geográfica en que ha de tener vigencia una determinada denominación. Así, y abusando de la terminología del derecho procesal, podemos decir que este apartado del reglamento debe definir claramente el ámbito de competencia territorial de la denominación.

En esta materia entonces, la actividad de peritos o expertos en la producción o elaboración de cada objeto de protección será especialmente relevante, pues serán ellos los encargados de informar hasta donde es posible extender las áreas de producción correspondientes a cada denominación. Asimismo, en esta tarea deben ser considerados al detalle todos los factores que permiten establecer la denominación, y, así, si las materias primas de un determinado producto se obtienen en una zona geográfica determinada, pero el proceso de elaboración se termina en otra, será necesario que el área de la denominación se extienda a ambas zonas.

5 Botana Agra, Manuel, Las Denominaciones de Origen, Tratado de Derecho Mercantil, Marcial Pons, Madrid, 2001, p. 100.

6 Cfr. idem, pp.101-105. 
En síntesis, esta delimitación geográfica determina en definitiva quiénes serán los titulares de la denominación, cuyo carácter colectivo beneficiará, en último término, a todos los productores con asiento en la zona geográfica que sirve de sustento a la misma.

Dependiendo del tipo de objeto amparado por la denominación, pensamos que esta zona puede ser de elaboración, producción, crianza, etc.

\section{c) Variedades o razas aptas para producir la materia prima}

En este punto nuevamente la voz de los expertos será determinante, pues como lo reconoce expresamente el proyecto de reforma de la Ley $\mathrm{N}^{\circ}$ 19.039 , la consideración de las materias primas es un aspecto del mayor interés al momento de definir la sustancia de la denominación de que se trate.

Al respecto, nuestra legislación sobre denominaciones de origen se ha vinculado siempre con la industria vitivinícola y, en tal sentido, ha existido una adecuada preocupación del legislador tendiente a establecer aquellas variedades de vid que son aptas para la elaboración de los productos protegidos. La misma preocupación, sin embargo, habrá de hacerse extensiva a todos aquellos productos susceptibles de ser protegidos bajo una denominación de origen, según venimos proponiendo en esta investigación.

En consecuencia, resulta indispensable contar una norma precisa que establezca cuáles son y qué características deben tener las materias primas que conformarán el objeto de protección, lo que presupone a su turno el determinar cuál será el origen geográfico de las materias primas permitidas para elaborar el producto amparado y qué porcentaje de dichas materias es considerado como mínimo para gozar de la protección ofrecida por la denominación.

\section{d) Procedimientos y prácticas de producción}

Este elemento de regulación debe considerar aquellas prácticas relacionadas con la elaboración, producción, crianza o cosecha tanto de las materias primas como de objetos mismos de la denominación.

El factor humano es determinante al momento de establecer una denominación de origen, y la forma en que se expresa con mayor fuerza se identifica precisamente con los procedimientos y técnicas usadas para desarrollar los productos protegidos por las denominaciones de origen.

En este mismo sentido debe ser considerado el lugar y la forma como se desarrollaran los procesos industriales ligados con los productos protegidos, así como también todo otro proceso complementario necesario para el desarrollo del producto final. 


\section{e) Producto final}

En caso que el objeto de protección no sea un producto agroalimentario neto, sino que se trate de un producto elaborado, es imprescindible determinar de manera precisa las características analíticas y organolépticas que permiten establecer que estamos frente al objeto de protección.

\section{f) Registros}

El reglamento de cada denominación debe establecer con precisión aquellos aspectos de la denominación que estarán sometidos a registro obligatorio, como por ejemplo: registro de productores, registro de predios, registro de instalaciones, registro de peritos reconocidos en determinadas materias, plantas envasadoras, distribuidores oficiales de materiales relacionados con la denominación, etc.

\section{g) Control}

Para el correcto funcionamiento de la denominación es necesario establecer un sistema de control al cual quedarán sometidos de manera obligatoria los productores que pretendan valerse de la denominación. Este sistema debe ser creado a priori por el reglamento, de forma que la incorporación de nuevos productores implique la aceptación de los términos de control establecidos.

El sistema de control debe ser transversal, esto es, aplicable a todas las actividades y productos ligados con la denominación en cuanto sean determinantes para la misma; así por ejemplo, proponemos que en el caso de una denominación que implique tiempo de guarda o maduración en recipientes de una calidad determinada, el sistema de control de la denominación permita impedir el uso de la misma por aquellos productores que hayan realizado la guarda o maduración en recipientes de una calidad distinta de la establecida.

\section{b) Derechos y obligaciones de los inscritos en la denominación}

El carácter colectivo de la denominación implica que los productores actuarán como un solo cuerpo, independiente de la organización que como persona jurídica se puedan dar bajo la normativa vigente. Lo anterior determina que el hecho de considerarse "miembro" de una denominación determinada implica asumir ciertas obligaciones y adquirir determinados derechos ligados con esta.

\section{i) Establecimiento de un Consejo Regulador}

Ni la legislación vigente ni la propuesta de modificación de la Ley $\mathrm{N}^{\circ} 19.039$ contemplan este tipo de organismo fiscalizador, indispensable 
- a nuestro entender- para el correcto funcionamiento de las denominaciones de origen. Por ello, y sin perjuicio de lo que se dirá más adelante, el reglamento debe contemplar el establecimiento de un consejo regulador.

\section{j) Infracciones $y$ sanciones}

Por último, el reglamento respectivo debe contener normas sobre funcionamiento interno y comportamiento ético-comercial de sus miembros, amén de las sanciones que deriven de la infracción de las mismas.

\section{B) Consejo Regulador de la denominación de origen}

Como lo anticipáramos, ni el proyecto de reforma de la Ley $N^{\circ} 19.039$, tantas veces citado, ni la legislación vigente, contemplan el establecimiento de una institución específica encargada de la tutela y supervisión de todas las materias y actividades vinculadas a las denominaciones de origen. La institución que tradicionalmente se ha ligado en nuestra legislación al tema de las denominaciones de origen ha sido el Servicio Agrícola y Ganadero, órgano al cual se entregan facultades de fiscalización y registro de aquellos aspectos propios de las denominaciones vitícolas, únicas existentes en nuestro país hasta ahora.

La propuesta de un Consejo Regulador apunta entonces a la creación de una institución de actuación in situ, anclada en el centro de todo el sistema de las denominaciones de origen e integrado principalmente por los propios productores interesados en preservarlas.

\section{a) Naturaleza jurídica}

En cuanto a su naturaleza jurídica, la doctrina comparada ha estimado tradicionalmente que los Consejos Reguladores (C.R.) son órganos complejos, por cuanto de su estructura interna se proyectan una serie de componentes que cumplen funciones determinadas. En este sentido, y adoptando la terminología española ${ }^{7}$, en cada Consejo debiera proyectarse un órgano ejecutivo representado por el presidente del Consejo, o bien por un directorio en pleno con posibilidades de delegar en un gerente general o en gerentes por área, dependiendo de los requerimientos de la denominación.

Desde el punto de vista de su existencia como sujeto de derecho, el Consejo Regulador podría tener una existencia independiente por la vía de constituirse como persona jurídica de derecho privado sin fines de lucro. Otra posibilidad es que se presente como la estructura orgánica de

Cfr. López Benitez, Mariano, Las denominaciones de origen, Cedecs Editorial, Barcelona, 1996, pp. 144-151. 
la persona jurídica que en definitiva formen los productores amparados con la denominación.

\section{b) Estructura interna}

La estructura del consejo debiera contemplar la participación de todos los productores registrados en la denominación, a través de un Consejo Superior o Consejo Pleno que tenga a su cargo la toma de las decisiones más importantes que afecten a la denominación.

Asimismo, resulta necesario incorporar un órgano técnico de carácter consultivo y fiscalizador, que podría adoptar el nombre de Comité de Calificación u otro similar.

Por último, se pueden crear órganos específicos encargados de conocer y solucionar los conflictos generados con materias relacionadas a la denominación, con facultades para aplicar eventuales sanciones como consecuencia de los hechos que conociere y que implicaren violaciones a la normativa contemplada en el Reglamento de la denominación.

\section{c) Integración}

Sobre la conformación de los Consejos Reguladores, algunos países de Europa han adoptado el sistema de integración mixta, incorporando a los mismos funcionarios de la administración del Estado junto a los privados. A nuestro juicio, el Consejo Regulador debería estar integrado si no en su totalidad por privados, al menos mayoritariamente, pues resulta de primordial interés para los particulares el mantener y fortalecer la denominación que los favorece.

\section{d) Funciones}

En cuanto a las funciones del Consejo, resulta interesante considerar aquellas que le han sido asignadas a los Consejos Reguladores en España ${ }^{8}$, país de larga experiencia y tradición en esta materia. En base esto, las funciones que debiera tener todo Consejo Regulador son las siguientes:

\section{i) Control de producción}

Aquí deben quedar comprendidas a las facultades que corresponden al Consejo en orden a orientar, vigilar y controlar la elaboración y calidad de los productos beneficiados con la denominación.

8 Cfr. BOtana Agra, ob. cit. pp. 109-110. 
En virtud de esta función, y a través de los órganos o funcionarios correspondientes, el Consejo podrá solicitar antecedentes a los productores asociados a la denominación respecto de sus procesos de elaboración, así como del tipo y calidad de las materias primas y elementos empleados en dichos procesos.

En este mismo contexto, los Consejos Reguladores deben contemplar un sistema de vigilancia y control permanente, gestionado por medio de fiscalizadores o inspectores dotados de las facultades necesarias para verificar el cumplimiento de cada una de disposiciones del Reglamento de la Denominación.

\section{ii) Velar por el prestigio de la denominación}

Indudablemente, el Consejo en su carácter de ente representativo de los miembros de la denominación debe cuidar de esta tanto a nivel interno como internacional, pudiendo ejercer a nombre de aquellos todas las acciones y derechos tendientes a prevenir su mal uso o a sancionarlo, según el caso.

\section{iii) Certificación de productos amparados por la denominación}

Si bien el sistema de una denominación de origen se basa en el origen geográfico de los productos, tal elemento no es suficiente por sí solo para una correcta administración de la denominación. La función propuesta pretende entonces que sea también el Consejo la institución oficial encargada de certificar los productos que, habiendo sido elaborados en el área geográfica de la denominación, pueden gozar de la misma por reunir los demás elementos ya analizados en este trabajo. En la práctica, esto se traduce en la entrega de certificados numerados, sellos de calidad u otros medios idóneos para garantizar que se cumplen los estándares de la denominación respectiva.

\section{iv) Gestión de los recursos de la denominación}

El Consejo debe estar facultado para administrar todos los recursos que sean comunes a una respectiva denominación. En esta gestión podrá adquirir bienes, fijar cuotas en dinero a los asociados, cobrar las sanciones pecuniarias en que incurran los miembros de una denominación y, en fin, toda otra actividad que diga relación con la administración del patrimonio común de la denominación.

En virtud de esta facultad, el órgano que corresponda deberá rendir cuentas periódicas y estará impedido de comprometer el patrimonio común de la denominación, por sobre montos que determine el Reglamento sin la autorización del pleno de los asociados a la misma. 


\section{v) Representación de la asociación}

El Consejo debe ser el representante de todos los miembros de la asociación, para todo tipo de actuaciones ya sea en el ámbito público o privado. Especialmente, debe tener la representación judicial de los miembros titulares de la denominación, reunidos en su seno.

En general, el Consejo debe encargarse también de llevar todos los registros vinculados a la denominación y procurar la correcta promoción de la misma, además de todas aquellas funciones que le establezca el reglamento particular de cada denominación.

En síntesis, todas estas propuestas, explicadas en base a la experiencia de países altamente desarrollados en el campo de la propiedad industrial, miran a un adecuado manejo de las denominaciones de origen que puedan establecerse en el país.

\section{Denominaciones del nORTE de Chile}

No obstante los rigores geográficos y climáticos del norte de nuestro país, en él destacan varios productos agroindustriales que presentan las características necesarias para ser amparados con denominaciones de origen. Cabe advertir, en todo caso, que la propuesta que aquí hacemos debe ser objeto de un análisis multidisciplinario para determinar, en definiti$\mathrm{va}$, si los productos respectivos tienen efectivamente las condiciones para constituir una denominación.

\section{A) Queso de Huentelauquén}

En el contexto de la macrozona norte, uno de los productos lácteos más típicos y estrechamente relacionado con su entorno geográfico, es el queso de Huentelauquén. La asimilación del producto con su lugar de origen es tal, que dichos quesos no son conocidos en relación con un tipo o variedad específico, sino que son denominados exclusivamente por el nombre geográfico.

Además, destaca en relación con estos quesos el factor humano, preponderante en toda denominación, por cuanto los especiales procesos de elaboración determinan la producción de un queso mantecoso de muy buena calidad.

\section{B) Aceitunas del Huasco}

Como en el caso anterior, este producto ha logrado también una identificación plena con el lugar de origen. El factor humano se manifiesta en las técnicas de cultivo, cosecha y preparación de las aceitunas. 
En particular, la denominación de origen que se pudiere lograr en relación a este producto cobra gran importancia ante la perspectiva de fabricación de aceite de oliva, usando como materia prima las Aceitunas del Huasco, pues en tal caso debieran desarrollarse denominaciones para los aceites teniendo como fundamento la denominación existente para la materia prima.

\section{C) Limones de Pica}

Este especial cítrico, que más que limón corresponde a una especie de lima ácida llamada "Lima Sutil" (Citrus Aurantifolia Swing), se produce con especiales características en el oasis de Pica, I Región, en atención principalmente a factores climáticos ligados a tal lugar.

Si bien la producción de limones en Pica es limitada, la eventual ventaja que pudiese reportar en el mercado el origen geográfico de los mismos se pierde actualmente al existir, en otras regiones del país, cultivos de la misma variedad que se comercializan también bajo el nombre de "Limón de Pica" (pese a las marcadas diferencias que existen entre unos y otros).

\section{D) Aceitunas de Azapa}

Este caso es digno de un acabado estudio técnico por cuanto, de la totalidad de las aceitunas provenientes del Valle de Azapa, existe un importante porcentaje que correspondería a una especie local conocida como "Variedad Azapa". La importancia agregada que cobra esta eventual denominación está dada por las proyecciones industriales en relación con la fabricación de aceite de oliva.

Junto con estos casos, en la zona norte destaca la producción de quesos de cabra en distintas localidades, así como también el cultivo con especiales características de las chirimoyas y papayas en La Serena.

\section{Bibliografía Citada}

- Decreto $N^{\circ} 16$ del Ministerio de Relaciones Exteriores

- Botana Agra, Manuel: Las Denominaciones de Origen. Tratado de Derecho Mercantil, Marcial Pons, Madrid, 2001,

- LÓPez Benítez, Mariano: Las denominaciones de origen, Cedecs Editorial, Barcelona, 1996. 